\title{
Nonequilibrium transient phenomena in the washboard potential
}

\author{
Chungho Cheng, ${ }^{1}$ Matteo Cirillo, ${ }^{2}$ Gaetano Salina, ${ }^{3}$ and Niels Grønbech-Jensen ${ }^{4}$ \\ ${ }^{1}$ Department of Mechanical and Aerospace Engineering, University of California, Davis, California 95616, USA \\ ${ }^{2}$ Dipartimento di Fisica and MINAS-Lab, Università di Roma "Tor Vergata”, I-00133 Roma, Italy \\ ${ }^{3}$ Istituto Nazionale di Fisica Nucleare, Sezione Roma “Tor Vergata,” Via della Ricerca Scientifica 1, I-00133 Roma, Italy \\ ${ }^{4}$ Department of Mathematics and Department of Mechanical and Aerospace Engineering,
}

University of California, Davis, California 95616, USA

(Received 21 February 2018; published 31 July 2018)

\begin{abstract}
Transient properties of the one-dimensional washboard potential are investigated in order to understand observed modulations in the statistics of escape events. Specifically, we analyze the effects of different kinds of initial conditions on the escape distribution obtained by linearly increasing the tilt of the potential. Despite the complexity of the dynamics leading up to the eventual escape, we find that the overall statistics can be interpreted in terms of the system parameters, which offers illuminating perspectives for driven one-dimensional systems with washboard potentials. We choose parameters sets relevant for Josephson junctions, a commonly studied system due to both its applications and its use as a model system in condensed matter physics.
\end{abstract}

DOI: 10.1103/PhysRevE.98.012140

\section{INTRODUCTION}

The washboard potential has been investigated for decades due to its relevance for fundamental aspects of general physics, condensed matter, Josephson effect, and superconducting electronics [1,2]. This one-dimensional potential, in reduced units, can be written

$$
U(\varphi)=(1-\cos \varphi)-\eta \varphi .
$$

For Josephson physics, $\varphi$ is the phase difference between the macroscopic wave functions of the two superconductors defining the junction. The potential $U(\varphi)$ is normalized to the characteristic Josephson energy $H_{J}=\frac{\Phi_{0} I_{c}}{2 \pi}$, with $\Phi_{0}=2.07 \times$ $10^{-15} \mathrm{~Wb}$ being the flux quantum and $I_{c}$ being the limiting (critical) superconducting current that the junction can support. The normalized bias current $\eta$ flowing through the junction is measured in units of the critical current $I_{c}$ and is the term responsible for tilting the cosine term giving the potential its characteristic shape reminiscent of a washboard [see Fig. 1(a)]. The potential barrier between a local minimum and the lowest of a neighboring maximum is $\Delta U=2\left(\sqrt{1-\eta^{2}}-\eta \cos ^{-1} \eta\right)$, and this barrier vanishes as $\eta$ becomes unity, facilitating the transition from the trapped state (zero voltage) to a ballistic state (nonzero voltage). The potential (1) was first suggested for Josephson systems by Anderson [3], but it is, of course, well known in general physics from the Newtonian dynamics of a forced pendulum in a gravitational field [4].

Thermal noise may induce the transition from zero to nonzero-voltage states for values of $\Delta U>0$ (and $\eta<1$ ) as originally investigated by Kurkijärvi [5] for the potential (1) using Kramers thermodynamic analysis [6]. Since the seminal work of Kurkijärvi, many studies have been devoted to the thermodynamics of the washboard potential through the prescribed experimental recipe of "adiabatically" increasing the bias current through the junction (usually a linear ramp increase in time) starting from the zero-voltage Josephson current, until a nonzero-voltage state is observed [7]. The sweep rate (current increase per second, $\mathrm{A} / \mathrm{s}$ in SI units), in this kind of experiment plays a paramount role and represents the speed at which the washboard potential is tilted downwards: In typical plasma frequency normalized units $[1,2]$ it is $\sigma=\frac{d \eta}{d t}$. The statistical distribution of the switching values of the bias current for which the transitions from the Josephson supercurrent (zero voltage) to a voltage state occur provides information about the sample temperature and potential barrier height [5]. The sweep rate, however, is a delicate parameter since the adiabatic (slow) limit [7] yields a narrow distribution for most relevant temperatures, thereby providing only limited information in regions of low switching probability. Increasing the sweep rate can provide a broader switching distribution, but as the sweep rate is increased and the loss parameter is kept very low, there is evidence from preliminary simulations that the plain Kramers model cannot be consistent with the acquired data.

Most of the past work has focused on sweep rates high enough to yield statistical information, yet low enough to not significantly enter into the nonequilibrium dynamics of the effects of the changing bias current. It has been speculated, however, that probing the nonequilibrium behavior of the washboard potential with high sweep rates can reveal qualitatively different information about the potential and its properties [8]. Further, the search for low dissipation digital devices based on the Josephson effect can benefit from information about the response of unshunted junctions subjected to very fast drives or clock excitations [9], which probe the dynamics as much as the equilibrium system properties. The interest in the development of single photon detectors [10] also requires a systematic investigation of the properties of Josephson dynamics and potentials spanning the properties in wide ranges of the parameters. We therefore investigate the intriguing response of the washboard potential when a very fast bias current sweep rate acts on the junction. We find that the statistical result from "nonadiabatic" bias sweep rates 

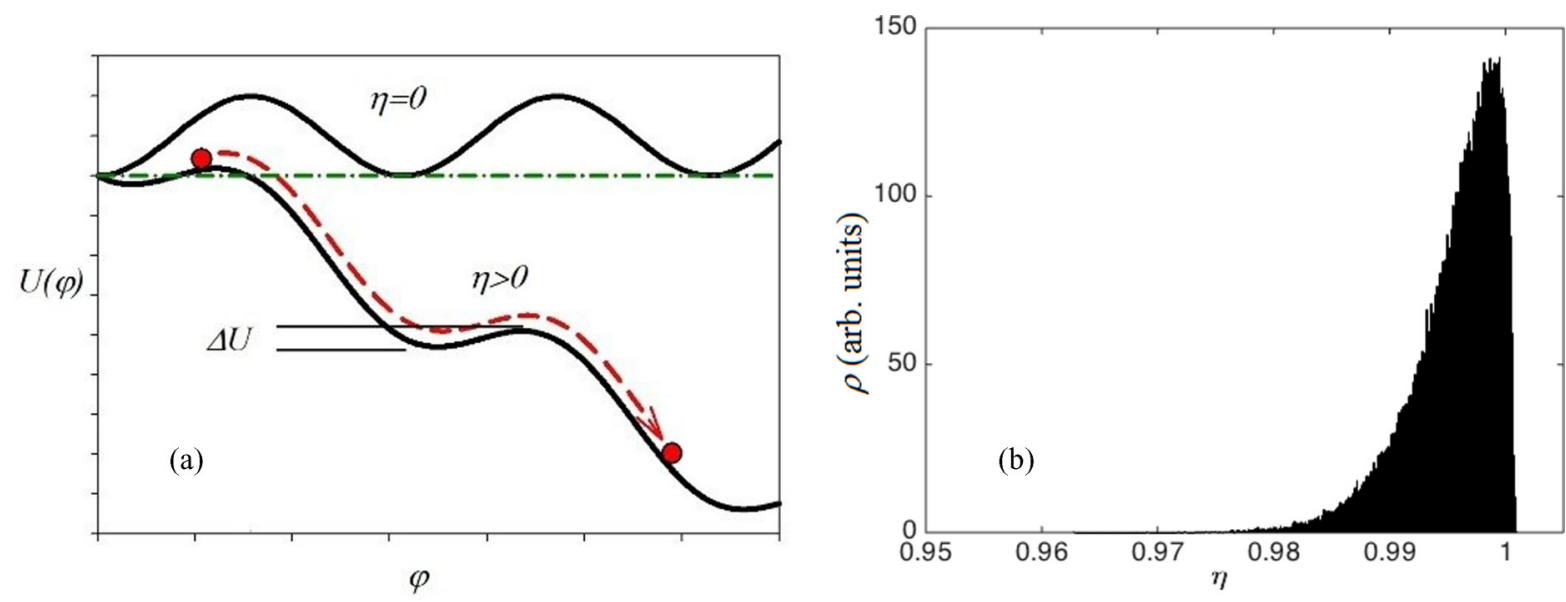

FIG. 1. (a) Sketch of the Josephson washboard potential given in Eq. (1); (b) a "typical" switching current distribution obtained from Eq. (2), setting as parameters loss factor $\alpha=10^{-4}$ and $\sigma=4 \times 10^{-4}$, normalized temperature $E_{N}=\frac{k_{B} T}{H_{j}}=10^{-3}$. The initial angle and the angular velocity, respectively, $\varphi_{0}(t=0)$ and $\dot{\varphi}(t=0)$ are both set to zero.

can be phenomenologically understood despite the complex dynamics.

The remainder of the paper is structured as follows: In the next section we describe the model equations and the techniques and approach employed for the integration; in Sec. III we describe the obtained results in four subsections, respectively, for simulations performed varying sweep rate, initial conditions, loss parameter, and temperature; in Sec. IV we summarize the paper and outline the conclusions.

\section{MODEL AND NUMERICAL APPROACH}

We model the dynamics of the thermal system with the potential (1) through the following Langevin equation,

$$
\ddot{\varphi}+\alpha \dot{\varphi}+\sin \varphi=\eta(t)+n(t),
$$

where time is normalized to the inverse of the Josephson junction angular velocity $\omega_{j}=\sqrt{\frac{2 \pi I_{c}}{\Phi_{0} C}}$ and $\alpha=\sqrt{\frac{\Phi_{0}}{2 \pi C R^{2} I_{c}}}=$ $\frac{1}{\sqrt{\beta_{c}}}$ is a dimensionless dissipation coefficient related to the McCumber parameter $\beta_{c}$ [1]. $C$ and $R$ are the capacitance and resistance of the junction, respectively; while $C$ is determined by the capacitance originated by the two electrodes separated by the thin oxide generating the junction, the resistance $R$ is usually associated with single electron or quasiparticle tunneling. Thermal fluctuations $n(t)$ are uniquely linked to the dissipation through the fluctuation-dissipation relationship [11]: $\langle n(t)\rangle=0$ and $\left\langle n(t) n\left(t^{\prime}\right)\right\rangle=2 \alpha \frac{k_{B} T}{H_{j}} \delta\left(t-t^{\prime}\right)$, where $T$ is the thermodynamic temperature of the system and $k_{B}=1.38 \times 10^{-23} \mathrm{~J} / \mathrm{K}$ is Boltzmann's constant. We integrate Eq. (2) numerically by a thermodynamically sound variant of the velocity-explicit Størmer-Verlet algorithm [12] with a normalized time step $\Delta t=0.02$, which is far below the stability limit of the integrator and well within the range of the time steps that produce statistically reliable simulation data for this problem and this method [13].

In order to simulate escape events from the potential well (1) we ramp the bias current from $\eta=0$, setting a linear time dependence for $\eta=\sigma t$, such that the system is unbiased at $t=0$, and record the distribution-of-currents values where the system switches to a voltage state. This procedure is repeated 30000-100000 times in order to obtain a statistically sound distribution. Our switching criterion is that the angle $\varphi>\varphi_{\text {esc }}$ where $\varphi_{\text {esc }}=\pi / 2$. When the escape angle is exceeded the system enters a rotating state in which the phase angle rapidly increases and generates, in Josephson junction terms, a dc-voltage state. For relatively high values of the sweep rates it can happen that the current $I$ of the ramp drives the particle slightly above the maximum Josephson critical current $(\eta>1)$ and therefore the just-mentioned condition for the switching angle would not make sense. However, in these cases we found that adopting as a criterion $\varphi>\varphi_{\mathrm{esc}}=\pi / 2$ also provided a very accurate condition for detecting a switch to a voltage state. Figure 1(b) shows a typical result of the statistical distribution of currents obtained for the parameter set: $\varphi(t=0)=\varphi_{0}=0$, $\dot{\varphi}(t=0)=\dot{\varphi}_{0}=0, \alpha=10^{-4}$, sweep rate $\sigma=10^{-4}$, and normalized temperature $E_{N}=\frac{k_{B} T}{H_{j}}=10^{-3}$. We see that the result, for the considered parameter set, in spite of the relatively high sweep rate, is much in line with those reported in the past [7].

It has been found, however, that peculiar choices of the initial angle $\varphi_{0}$, for values of the ratio $\kappa=\alpha / \sigma$ below unity, can condition the maximum escape current of the washboard potential [14] even for $T=0$. As we shall see in the following section, thermal effects do not significantly change the observed modulation phenomena in the statistical current distributions of the escape process, and the observations are primarily due to initial conditions and the parameter $\kappa$ such that modulations in the distributions are observed for $\kappa \leqslant 1$.

\section{RESULTS}

\section{A. Effect of sweep rate}

Figure 2 shows simulated bias probability densities $(\rho)$ for switching events, i.e., the distribution of recorded values of the $\eta$ values for which switching occurs as the bias current is increased according to $\eta=\sigma t$. The system parameters are $\varphi(t=0)=\varphi_{0}=0.1, \dot{\varphi}(t=0)=\dot{\varphi}_{0}=0, \alpha=10^{-4}$, and normalized temperature $E_{N}=\frac{k_{B} T}{H_{j}}=10^{-3}$. In the sequence of Figs. 2(a)-2(d) we change the sweep rate $\sigma=\frac{d \eta}{d t}$ as follows: 

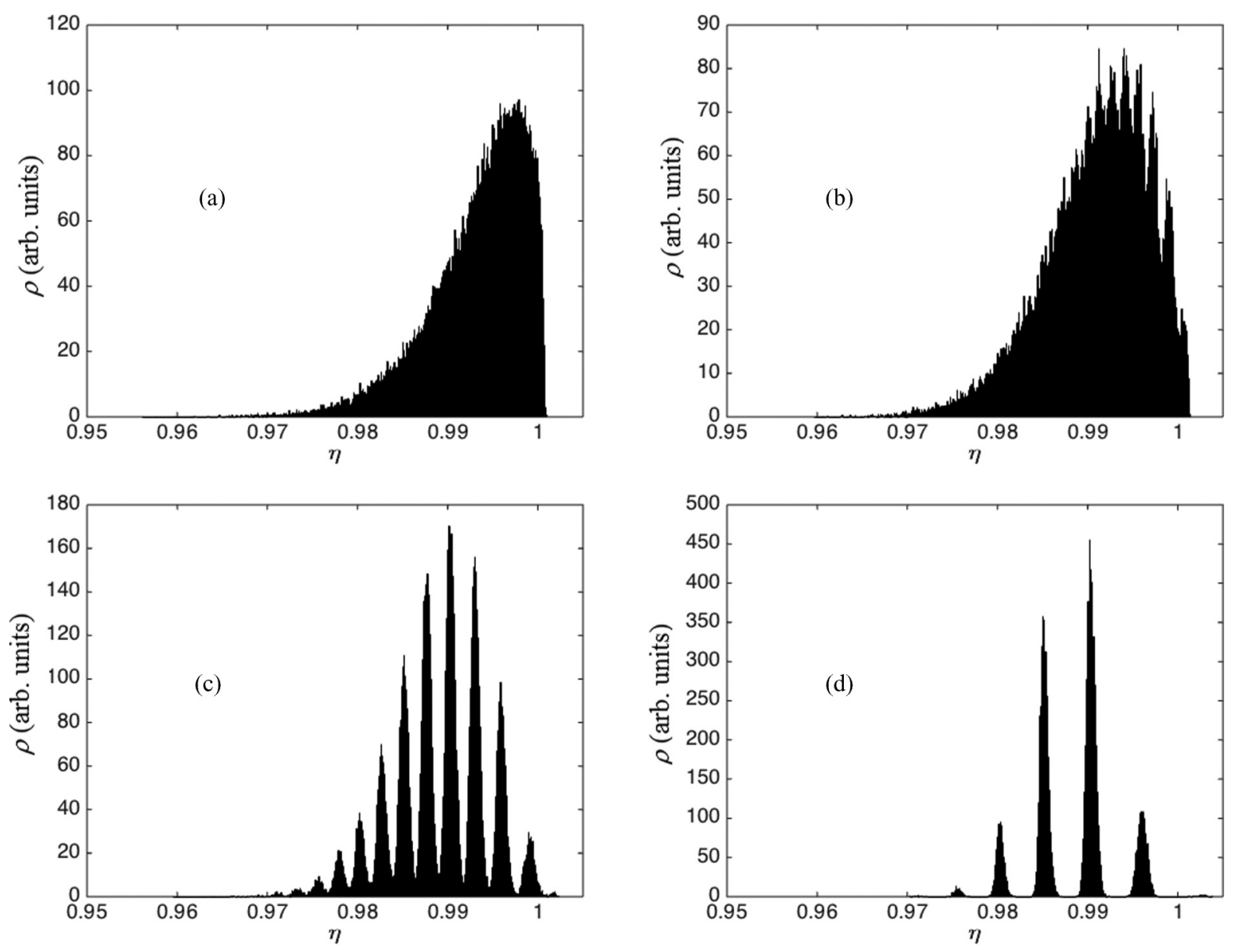

FIG. 2. Simulated probability density distributions of escape from the potential well as the tilt is increased, starting from $\eta(0)=0$. Parameters are $\dot{\varphi}(t=0)=\dot{\varphi}_{0}=0, \alpha=10^{-4}$, normalized temperature $E_{N}=\frac{k_{B} T}{H_{j}}=10^{-3}$. The specific parameter values for the four distributions are (a) $\varphi_{0}=0.1$ and $\sigma=5 \times 10^{-5}$, (b) $\varphi_{0}=0.1$ and $\sigma=10^{-4}$, (c) $\varphi_{0}=0.1$ and $\sigma=2 \times 10^{-4}$, and (d) $\varphi_{0}=0.1$ and $\sigma=4 \times 10^{-4}$.

(a) $\sigma=5 \times 10^{-5}$, (b) $\sigma=10^{-4}$, (c) $\sigma=2 \times 10^{-4}$, and (d) $\sigma=4 \times 10^{-4}$. Figures $2(\mathrm{a})-2(\mathrm{~d})$ show that a perturbed initiation of the sweep may induce resonant features in the switching distribution, and these features depend on the sweep rate. We remark that the plot of Fig. 1(b) was obtained for the same values of Fig. 2(b), but setting no initial "perturbation," i.e., $\varphi_{0}=0$ and $\dot{\varphi}_{0}=0$, which returned a standard switching current distribution.

Notice that the phenomenon of peaked distributions was observed and reported previously for a spatially extended system without in-depth analysis [15]. It is noticeable that the high end of the distribution may extend beyond the critical value $\eta=1$. This is due primarily to the fast sweep rate, and in part to the origin of the resonant features. The fast sweep rate is accelerating the potential well in the positive $\varphi$ direction, thereby delaying the system from reaching the state of free rotation [14]. The resonant features are due to initial condition induced plasma oscillations, which may be timed such that the switching probability is small when $\eta=1$ is reached, thereby giving rise to a delayed switching past $\eta=1$.

The normalized parameters chosen in the previous paragraph would correspond, for an all-niobium junction with a critical current of $10 \mu \mathrm{A}$ and a junction area of $100 \mu \mathrm{m}^{2}$, to a temperature of a few hundred milli-Kelvin and to a sweep rate of the order of $30 \mathrm{~A} / \mathrm{s}$ and above. This sweep rate is of the order of the highest so far reported in potential escape experiments [8], but as we argue below, our results may extend to lower sweep rates as well.

In the simulations we have found that peaks in the statistical distribution are exhibited when the parameter $\kappa=\frac{\alpha}{\sigma}$ is of the order of unity or below. This effect is made clear in Fig. 2 where we see that in (a), for $\kappa=50$, there is no evidence of peaks. These start being evident in (b) where $\kappa=1$, while in (c) and (d) where $\kappa$ is equal, respectively, to 0.5 and 0.125 the peaks are well spaced. We will see now that, when this condition on $\kappa$ is satisfied, for a different type of realistic initial condition, the response can still show, for adequate temperature choice, peaked statistic distributions like those shown in Fig. 2.

\section{B. Effects of initial conditions}

Figures 3(a) and 3(b) show the results obtained for the same set of parameters, respectively, of Figs. 2(b) and 2(c) but with the initial condition $\varphi_{0}=-0.1$. The comparison shows that there is very little difference between the statistical distributions when inverting the sign of the initial angle. The only difference is that of an opposite phase, where low probability in a distribution generated by one sign of the initial 

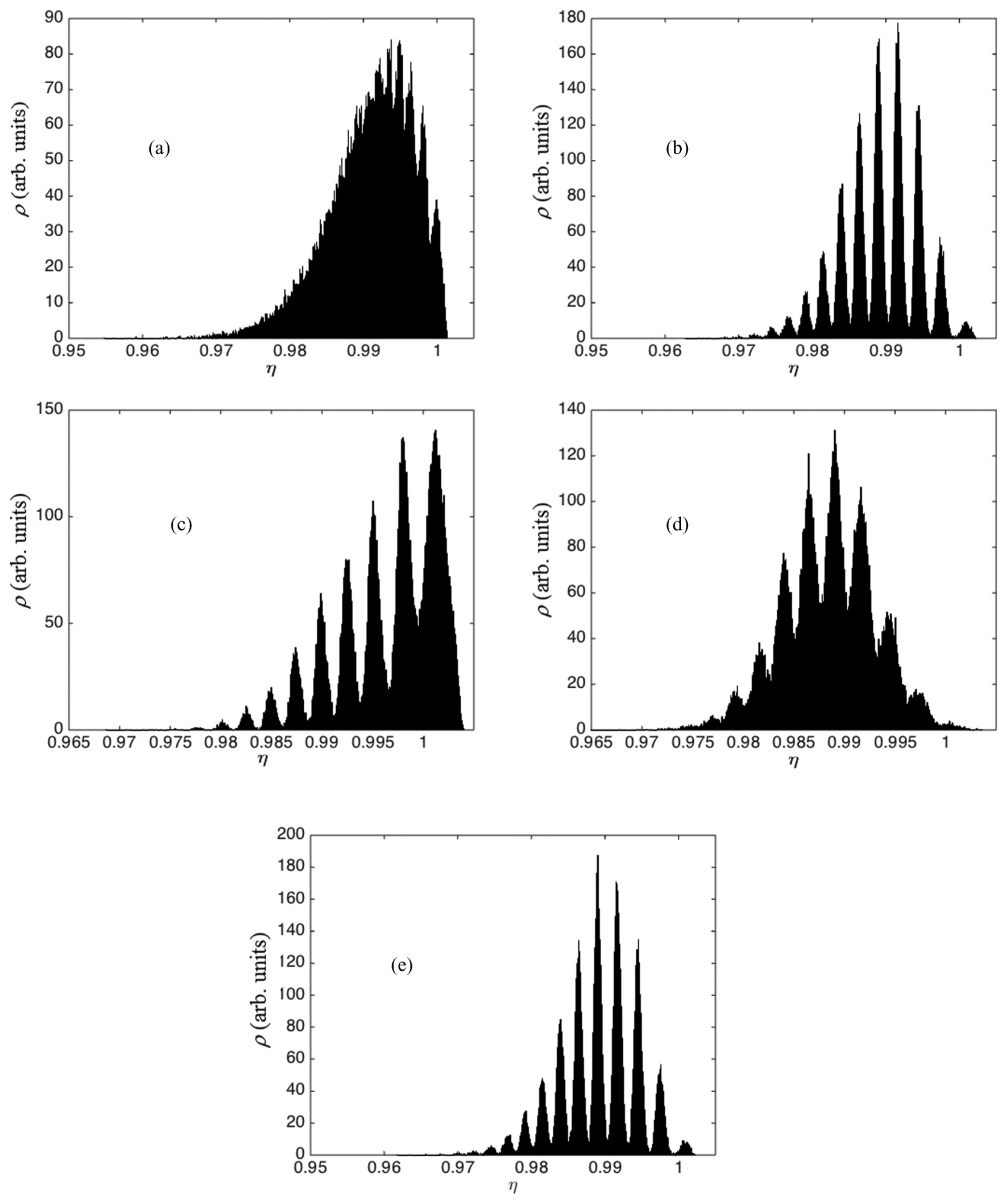

FIG. 3. Distributions for different initial conditions: (a), respectively (b), statistical distribution obtained for the same set of parameters as (b), respectively (c), of Fig. 2 but fixing $\varphi_{0}=-0.1$; (c) random phase in the interval $[-0.1,0.1]$ and zero velocity; (d) random phase in the interval $[-0.1,0.1]$ with the phase derivative determined from a constant energy constraint. For both (c,d) we have $\alpha=10^{-4}$ and $\sigma=4 \times 10^{-4}$. In (e) we show that with decreasing the integration time step by a factor of 2 , the result of the integration corresponding to the parameters reported in (b) is identical.

condition becomes high probability for the opposite sign in the initial condition and vice versa. This observation establishes the direct connection between the initial condition at zero bias current and the modulation of the switching distribution. The phenomenon is fully consistent with the dynamics of the switching which consists of attempts to overcome the potential barrier by the induced plasma oscillations [14]. More results confirming this basic conjecture are shown in the next paragraphs.

Figure 3(c) shows the result of simulations performed when choosing uniformly distributed random initial angles in the interval $[-0.1,0.1]$, for $\dot{\varphi}_{0}=0$. Figure $3(\mathrm{~d})$ shows the result 


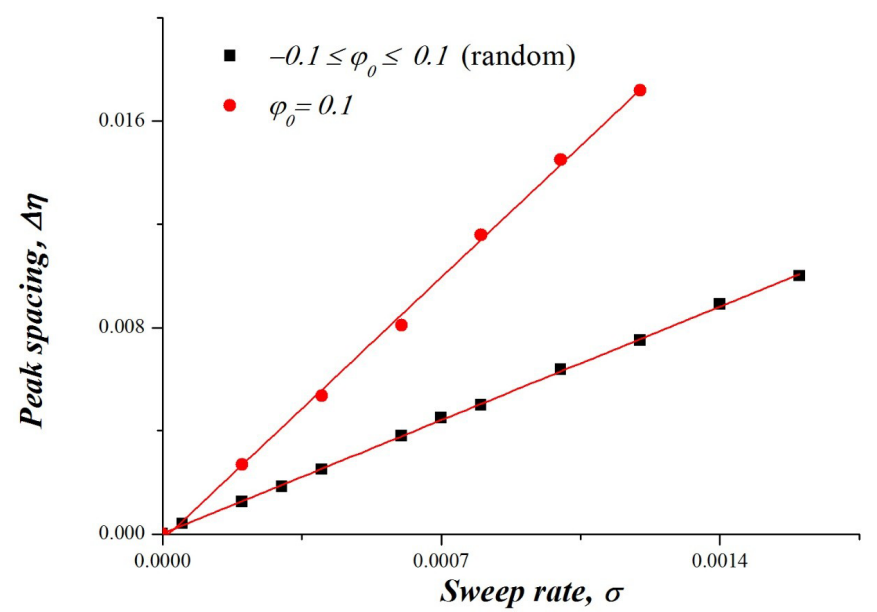

FIG. 4. Dependence of the peak spacing upon the sweep rate $\sigma$. Analyzing this dependence in terms of un-normalized quantities of this dependence we find a significant condition regulating the spacing of the peaks and the peculiar shape of the distributions. The two straight lines' dependencies are relative to different types of initial conditions.

when we set an initial random angle in the same interval, but with $\dot{\varphi}_{0}$ determined such that the energy of the initial condition is always the same, namely, $2 E_{0}=\dot{\varphi}_{0}^{2}+\varphi_{0}^{2}$ with $E_{0}=5 \times$ $10^{-3}$. For both Figs. 3(c) and 3(d) parameters are $\alpha=10^{-4}$, $\sigma=4 \times 10^{-4}$ and $E_{N}=\frac{k_{B} T}{H_{j}}=10^{-3}$. The modulating peaks will disappear when the ratio $\kappa>1$. As stated above, however, no peaklike features are observed for the initial condition at the minimum of the initial potential well. It is worth noting that $\kappa=$ $\frac{\alpha}{\sigma}$ has been invoked to characterize, from a different physical perspective, fast sweep experiments in Josephson junctions [8]. In Fig. 3(e) we verify the validity of the choice of the numerical time step $d t=0.02$ by comparing a distribution obtained for the same parameters as in Fig. 3(b) but with an integration time step $d t=0.01$.

We now investigate what determines the spacing between the observed peaks in the escape distributions. Given a specific value of the loss factor, we have found that the peak spacing, when the peaks appear, does not depend on $E_{N}$, but only on initial conditions and sweep rate $\sigma$. Figure 4 shows the measured peak spacing $(\Delta \eta)$ as a function of the applied sweep rate for two families of initial conditions. As indicated in the figure the squares are relative to distributions obtained setting the initial angle $\varphi_{0}$ randomly in the interval $[-0.1,0.1]$. The linear fit to the data in this case indicates that $\Delta \eta=2 \pi \sigma$ within an uncertainty on the proportionality constant less than a few parts over $10^{5}$. From the characteristic units this result indicates that $\Delta I_{P S} / t_{j}=(\Delta I / \Delta \tau)$, where $\Delta I_{P S}$ is the peak spacing in amperes, $\Delta I$ the step (in amperes) of the current ramp, $t_{j}=1 / \omega_{j}$ is the Josephson characteristic time, and $\tau$ is time (in seconds). Thus, the appearance of the peaks is related to the fact that the ratio between peak spacing and Josephson plasma time (akin to a "Josephson" time derivative of the current) equals the specific sweep rate, the current time derivative in SI units.

The circles in Fig. 4 represent the spacings obtained from the initial condition $\varphi_{0}=0.1$. We can see that the dependence of the peak spacing upon the sweep rate is linear and has a slope 14.54, which is more than a factor of 2 above that corresponding to the random distribution of the initial angle $(2 \pi)$ seen above. A reduction of a factor of 2 in the spacing can be expected if we hypothetically "merge" the results obtained for $\varphi_{0}=0.1$ [Fig. 2(c)] and $\varphi_{0}=-0.1$ [Fig. 3(b)]. Since these opposite conditions provide escape distributions which are out of phase, it is reasonable to expect that, taking the initial angle uniformly distributed in the interval $[-0.1,0.1]$, escape peaks at both the extremes of the interval will be recorded, implying therefore a difference of a factor 2 of the peak spacing.

We note that setting an initial angle of the order of one-tenth of a radian indicates that we are choosing an initial energy of the system equivalent to the thermal energy corresponding to a temperature of $1 \mathrm{~K}$ for a niobium junction described above. Moreover, the peak spacing that we observe would correspond, for typical Josephson parameters like those indicated before, to currents of the order of tens of nanoamperes. However, temperature might not be the only physical reason generating the initial disturbance.

It has been pointed out that the adiabatic approximation of the Kramers model would remain valid until the frequency of the periodic potential would hit that of proper modes of the junction [16,17]. The analysis of the previous paragraph is consistent with this prediction since it tells us that that the physical reason for generating the peaks in the escape probability distributions is essentially an interaction between the (high frequency) plasma oscillations generated by the initial conditions and ramp current increase. Thus for our "nonequilibrium" statistical distributions the transient oscillations act as a periodic modulation of the potential.

\section{Effect of loss parameter}

A key issue is the dissipation term in the commonly used pendulum or circuit model [2] which is represented as a linear, constant friction term. Josephson junctions are much more complex than such a simple model, and especially the quasiparticle tunneling mechanism is grossly oversimplified. The complexity of the dissipation issue can be appreciated from the more complete microscopic theory [18] in which tunneling of both superconducting and dissipative currents is mixed through frequency-dependent memory terms in an integrodifferential equation [19]. From this, one can appreciate that an effective phenomenological friction term for the nearzero-voltage states relevant for the investigations here is not straightforward to assess. Thus, we believe that a reasonable choice for such a simplified friction parameter in Eq. (2) must be rooted in simulation results that are consistent with experimental observations.

We now analyze the specific effect of dissipation on the statistical distribution. Figure 5 shows what happens, for a given sweep rate and temperature, when the loss parameter is decreased, while sweep rate and temperature are kept constant, $\sigma=4 \times 10^{-4}, E_{N}=10^{-3}$, and the initial angle is uniformly distributed in the interval $[-0.1,0.1]$ with $\dot{\varphi}_{0}=0$. From Fig. 5(a) to Fig. 5(b) the friction $\alpha$ is decreased from $1.6 \times 10^{-3}$ (a) to $10^{-4}$ (b). It is clear that decreasing the dissipation enhances the fidelity of the peaks, and still the effect becomes pronounced for $\kappa \leqslant 1$. We have also analyzed the effect of the dissipation on each single peak and the result is shown in 

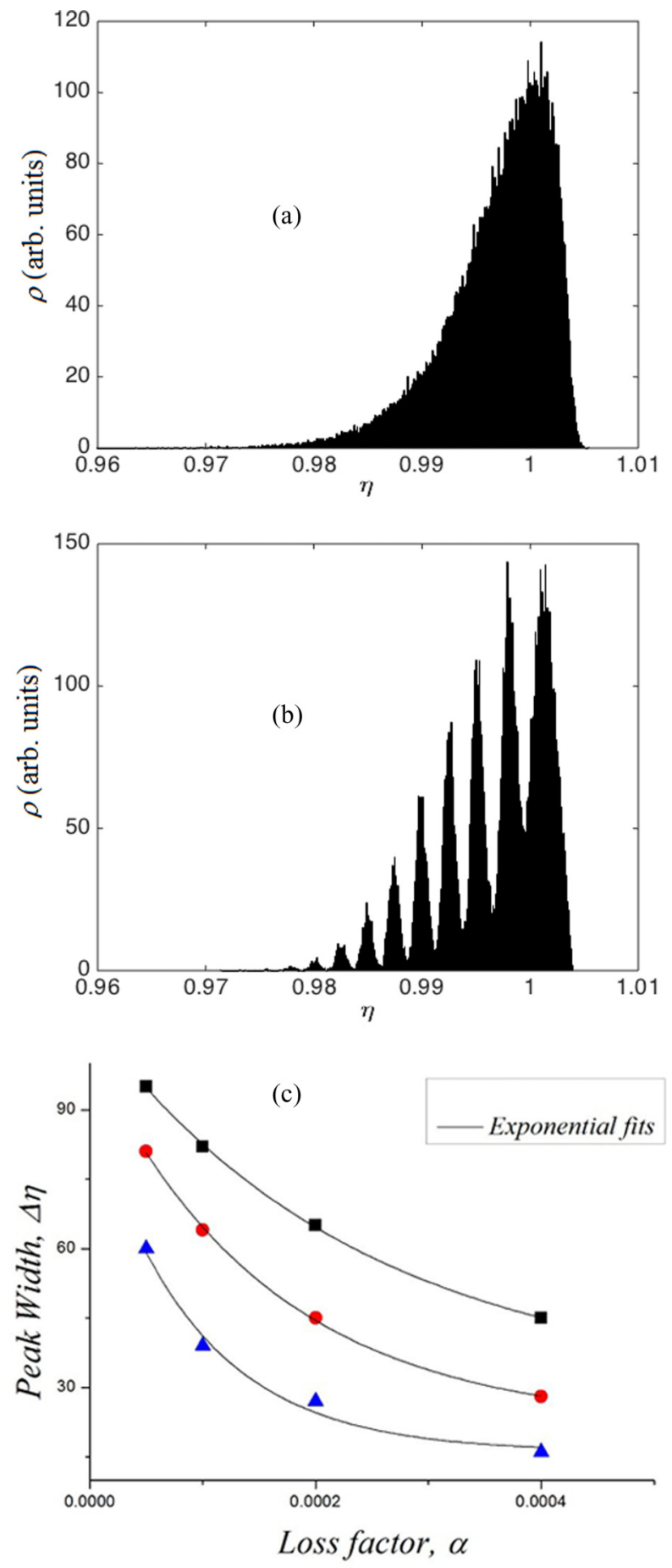

FIG. 5. Effect of loss on the statistical distribution. Here $\sigma=4 \times$ $10^{-4}$ while the loss parameter $\alpha$ is, respectively, $1.6 \times 10^{-3}$ and $10^{-4}$ for $(a, b)$. The loss parameter is responsible for the width of the peaks which depends indeed exponentially upon it, as shown in (c).

Fig. 5(c), where we show the width of the peaks as a function of $\alpha$. The widths of the peaks appearing at given values of the current bias $\eta$ were measured for different values of $\alpha$. The exponential curves are fitted to the data, and we thus conclude that the dependence of the peak width on $\alpha$ is exponential. The loss factor does not influence peak spacing, but when it becomes too high it induces a widening which will diminish
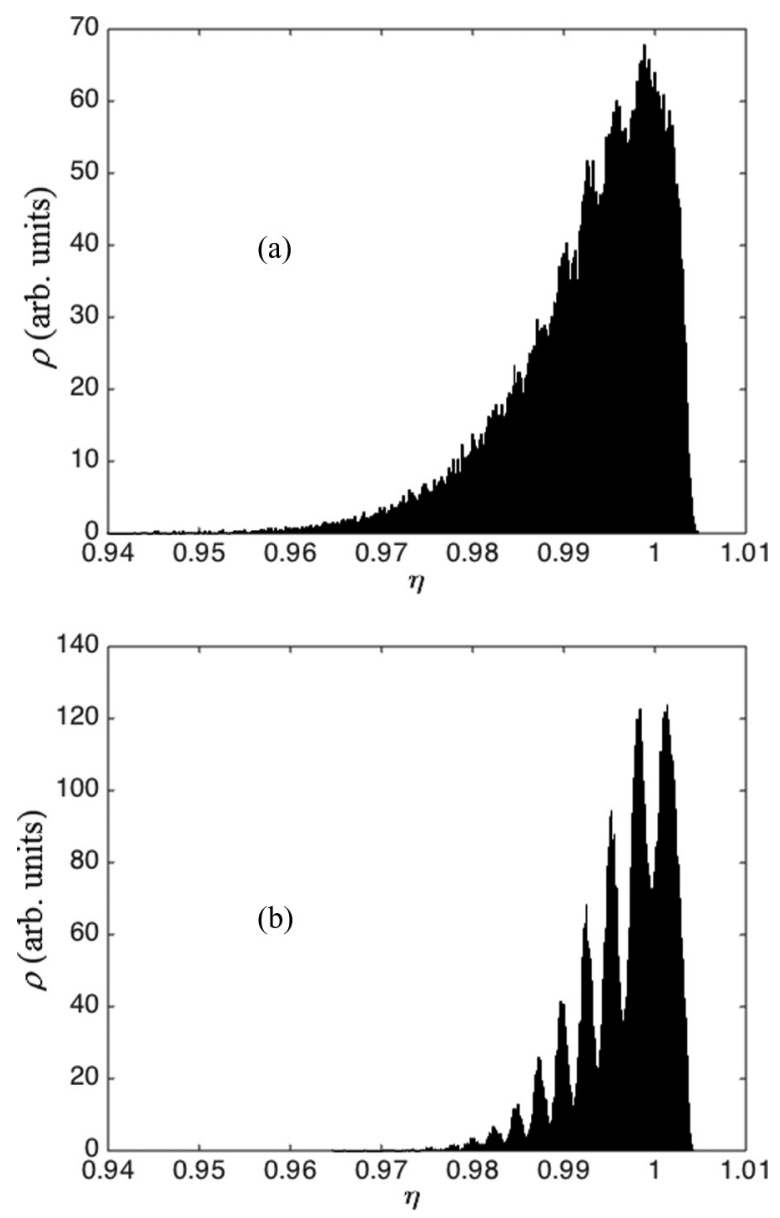

FIG. 6. Effect of the temperature on the peaked escape distributions for fixed values of the other parameters $\left(\alpha=2 \times 10^{-4}\right.$ and $\left.\sigma=4 \times 10^{-4}\right)$; in (a,b) we set $E_{N}$ equal, respectively, to $4 \times 10^{-3}$ and $10^{-3}$. In experimental units going from (a) to (b) could mean, for typical Josephson parameters, stepping from a range of temperature of a few Kelvin to the hundreds of milli-Kelvin range.

the resolution and prevents the observation of peaks. This is entirely consistent with the behavior of a damped oscillator.

Interest for the fast sweep conditions has been reported by analyses performed in the overdamped limit imposing periodic potentials $[16,17]$. In spite of these different dynamical conditions in this previous work evidence of peaked statistical distributions might be inferred from the reported data: a derivative of the escape probability of the plots shown in Figs. 3 and 7 of Ref. [16] and Fig. 1 of Ref. [17] would return peaked probability distributions. Moreover, in these papers the role of the escape time in the production of the statistical distributions was also investigated and our conclusion relating the Josephson oscillation time to the characteristic sweep times, as extracted from the straight line of Fig. 4, fits those previous observations; all are consistent with the discussion at the end of the previous subsection.

\section{Effect of temperature on statistical distributions}

The modulating peaks on the switching distributions may also depend on temperature, and Fig. 6 shows some results of the numerical integration. The simulations have been 

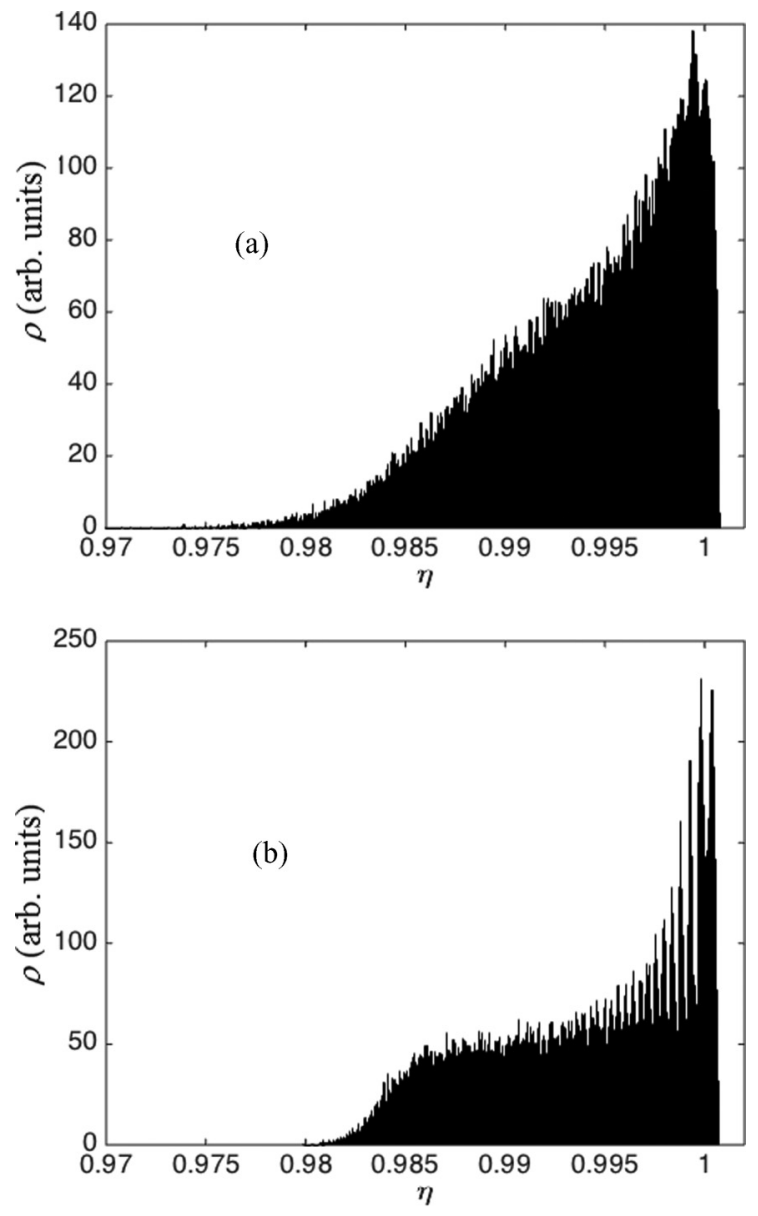

FIG. 7. An example of current distribution for sweep rate $\sigma=$ $5 \times 10^{-5}$ but for loss factor $\alpha$ equal to $10^{-5}$ (a) and $10^{-6}$ (b). The thermal energy $E_{N}=10^{-3}$. As we see, with scaling down the sweep rate and loss the distributions still become peaked for $\alpha / \sigma$ less than 1.

conducted for $\sigma=4 \times 10^{-4}$ and $\alpha=2 \times 10^{-4}$ while the normalized temperatures $E_{N}=4 \times 10^{-3}$ and $E_{N}=10^{-3}$ have been chosen for (a) and (b), respectively. For the typical niobium junction parameters mentioned in Sec. III A this would roughly correspond to $4 \mathrm{~K}$ and $1 \mathrm{~K}$. We can clearly see how the appearance of multipeaked distributions is produced for the lower values of the temperature (provided that $\kappa \leqslant 1$ ), exemplified in Fig. 6 using a random initial angle uniformly chosen in the interval $[-0.1,0.1]$, along with $\dot{\varphi}_{0}=0$.

We noted previously that the sweep rates we have studied here are higher than the highest reported in experiments [8]. However, we also stated that the results could be scaled to lower sweep rates. Plots analogous to those shown in Figs. 2-6 can be obtained, for example, by choosing $\sigma=5 \times 10^{-5}$ and setting as a consequence an appropriately low value for the dissipation: The result in this particular case is shown in Fig. 7 where in (a) we have $\alpha=10^{-5}$ while in (b) we have $\alpha=10^{-6}$, and we see that the peaked distribution is generated for the lower of the two values. In Fig. 7 we have imposed initial conditions as in Fig. 6, and a temperature of $E_{N}=10^{-3}$. We note that $10^{5}$ switching events were used to generate Fig. 7. In order to simulate cases with lower sweep rates one needs to improve the resolution on the current (horizontal) axes of the statistical distributions, implying that the number of switching events must be increased further, resulting in substantially increasing simulation time.

\section{CONCLUSIONS}

The results presented above rely on the fact that specific initial conditions imposed at the initiation of each sweep of the escape process out of a Josephson junction washboard potential give rise to underdamped oscillations. These oscillations survive coherently throughout the sweep until the switching event takes place, similar to other reported cases [2,20]. We presume that these oscillations are generated by an initial perturbation due to temperature, or recurring cycling of the current sweeps or the perturbation from the onset of the bias sweep itself. The last two processes both have the possibility to induce initial energies in excess of the thermodynamic temperature. Additionally, with a loss parameter small enough for the dissipation timescale to be lower than the time it takes to complete a bias sweep, the coherence of an initial perturbation can provide the plausible plasma oscillation phase timing during the possible switching regime. The transient high frequency oscillations have the same effect on the escape process of a periodic drive $[16,17]$ and therefore the nonequilibrium effects can be observed in the statistic distribution of the escape process.

Direct simulations and details of the switching process will be addressed in a future publication [14]. Initial conditions represent a key factor in the behavior of dynamical systems and those chosen for the present investigation have been motivated by the temperature of the system and possible dynamical "history." We have shown that the initial disturbances can create coherent oscillations that result in modulated switching distributions for low dissipation and high sweep rates and found that the ratio $\kappa=\alpha / \sigma$ plays a key role in the observed phenomenon. It is clear that a necessary condition for observing modulated and peaked switching distributions is that this ratio must be close to unity or below regardless of what (realistic) initial conditions are imposed. The interplay of temperature, dissipation, and time-dependent forcing excitations can be traced in terms of other parameters of the model equation. The analysis presented in this paper is based almost exclusively on numerical simulations and not on commonly used perturbation methods for driven-damped pendulum equations. The reason is that, while the phenomenon is intuitively well described by the numerical investigation, it is difficult to apply perturbation techniques near the switching current since that value is close to unity, and therefore associated with mathematical singularities. Thus, while we have not provided direct analytical expressions for, e.g., specific phase relationships between initial conditions and locations of modulation peaks in the resulting distributions, we believe that our analysis can provide valuable information and intuition for both Josephson systems and other classes of potentials that are similarly perturbed.

\section{ACKNOWLEDGMENTS}

M.C. and G.S. acknowledge partial support for the present work from INFN (Italy) under project FEEL. We thank our colleague James A. Blackburn for helpful comments and suggestions. 
[1] A. Barone and G. Paternò, Physics and Applications of the Josephson Effect (John Wiley, New York, 1982), p. 143; T. Van Duzer and C. W. Turner, Principles of Superconducting Devices and Circuits (Prentice-Hall, Englewood Cliffs, NJ, 1999), p. 204.

[2] J. A. Blackburn, M. Cirillo, and N. Grønbech-Jensen, Phys. Rep. 611, 1 (2016).

[3] P. W. Anderson, Special effects in superconductivity, in Lectures on the Many Body Problem, edited by E. R. Caianiello (Academic Press, New York, 1964), Vol. 2, pp. 113-135.

[4] G. L Baker and J. A. Blackburn, The Pendulum, A Case Study in Physics (Oxford University Press, Oxford, 2005).

[5] J. Kurkijärvi, Phys. Rev. B 6, 832 (1972).

[6] H. A. Kramers, Physica 7, 284 (1940).

[7] T. Fulton and L. Dunkelberger, Phys. Rev. B 9, 4760 (1974); M. Büttiker, E. P. Harris, and R. Landauer, ibid. 28, 1268 (1983); J. M. Martinis, M. H. Devoret, and J. Clarke, ibid. 35, 4682 (1987); M. G. Castellano, G. Torrioli, C. Cosmelli, A. Costantini, F. Chiarello, P. Carelli, G. Rotoli, M. Cirillo, and R. L. Kautz, ibid. 54, 15417 (1996).

[8] P. Silvestrini, V. G. Palmieri, B. Ruggiero, and M. Russo, Phys. Rev. Lett. 79, 3046 (1997); A. Barone, R. Cristiano, and P. Silvestrini, J. Appl. Phys. 58, 3822 (1985).

[9] J. Ren, V. K. Semenov, Y. A. Polyakov, D. V. Averin, and J. S. Tsai, IEEE Trans. Appl. Superconductivity 19, 961 (2009); O. A. Mukhanov, ibid. 21, 760 (2011); M. Lucci, Jie Ren, S.
Sarwana, I. Ottaviani, M. Cirillo, D. Badoni, and G. Salina, ibid. 26, 100905 (2016).

[10] G. Oelsner, L. S. Revin, E. Il'ichev, A. L. Pankratov, H.-G. Meyer, L. Grönberg, J. Hassel, and L. S. Kuzmin, Appl. Phys. Lett. 103, 142605 (2013); G. Oelsner, C. K. Andersen, M. Rehák, M. Schmelz, S. Anders, M. Grajcar, U. Hübner, K. Mølmer, and E. Il'ichev, Phys. Rev. Appl. 7, 014012 (2017).

[11] G. Parisi, Statistical Field Theory (Addison-Wesley, Reading, MA, 1988).

[12] N. Grønbech-Jensen and O. Farago, Mol. Phys. 111, 983 (2013).

[13] E. Arad, O. Farago, and N. Grønbech-Jensen, Isr. J. Chem. 56, 629 (2016).

[14] G. Salina, M. Cirillo, M. Lucci, C. Cheng, N. Grønbech-Jensen, and J. A. Blackburn (unpublished).

[15] N. Grønbech-Jensen and M. Cirillo, Phys. Rev. B 70, 214507 (2004); see Fig. 7 of this paper in the high sweep rates region.

[16] A. L. Pankratov and M. Salerno, Phys. Rev. E 61, 1206 (2000).

[17] A. L. Pankratov and M. Salerno, Phys. Lett. A 273, 162 (2000).

[18] N. R. Werthamer, Phys. Rev. 147, 255 (1966); U. K. Poulsen, Ph.D. thesis, The Technical University of Denmark, 1973.

[19] N. Grønbech-Jensen, S. A. Hattel, and M. R. Samuelsen, Phys. Rev. B 45, 12457 (1992).

[20] J. E. Marchese, M. Cirillo, and N. Grønbech-Jensen, Phys. Rev. B 73, 174507 (2006). 